\title{
Präventionsgeschichte als Kulturgeschichte der Gesundheitspolitik
}

MARTIN LENGWILER, JEANNETTE MADARÁSZ

1983, auf dem Höhepunkt des Kalten Krieges, äußerte sich die britische Sozialanthropologin Mary Douglas in ironischer Zuspitzung zur Risikowahrnehmung der amerikanischen Bevölkerung: „What are Americans afraid of? - Nothing much really, except the food they eat, the water they drink, the air they breathe [...].“ (Douglas 1983: 10) In einer Zeit, die vom Wettrüsten, einer schlechten Wirtschaftslage und horrenden Staatsdefiziten geprägt war, drehten sich die Alltagssorgen Amerikas nicht um die großen politischen und wirtschaftlichen Krisen der Zeit, sondern um banale Ernährungs- und Trinkgewohnheiten. Zweierlei ist bemerkenswert am Kommentar von Douglas. Er verweist einerseits auf die Alltäglichkeit moderner Risikovorstellungen und der damit verbundenen Präventionspraktiken. In der Tat haben gesundheitspolitische Popularisierungen und pathologisierende Formen der Zivilisationskritik in vielen westlichen Ländern dazu geführt, dass im 19. und 20. Jahrhundert überlieferte Formen des Essens und Trinkens problematisiert, aufgebrochen und zum Gegenstand eines gesundheitsorientierten Präventionsdiskurses gemacht wurden.

Andererseits illustriert das Zitat von Douglas, dass die Transformation präventiver Verhaltensweisen in historischer Perspektive ein vielschichtiges Phänomen ist. Nicht ohne Grund ist die Geschichte der modernen Gesundheitsprävention noch weitgehend ungeschrieben. Denn ihr haftet etwas Unwirkliches, Unauffälliges an. Unwirklich, weil Prävention einen Schaden antizipiert, der meist gar nicht eintritt, weil er vorsorglich verhindert werden soll. Und unauffällig, weil Prävention meist die Ebene unspektakulären Alltagsverhaltens (Essen und Trinken, 
Rauchen, Bewegung etc.) betrifft und dabei oft in wenig sichtbaren Infrastrukturbereichen ansetzt, etwa bei der Nahrungsmittelhygiene, der Fabrikinspektion oder allgemein der Hygienisierung des öffentlichen Raumes. Zwar hat das Thema Gesundheitsprävention in den letzten Jahren politisch und gesellschaftlich an Bedeutung gewonnen. Trotzdem ist es keineswegs selbstverständlich, Prävention zu einem Gegenstand der historischen Forschung zu machen.

Dieses Forschungsdesiderat bildete den Ausgangspunkt des vorliegenden Sammelbandes. Die folgenden Beiträge verstehen sich als exploratives Unternehmen, das einige zentrale Schauplätze und Schlüsselmomente einer Präventionsgeschichte der Moderne in den Blick nimmt. Die Entwicklung der Gesundheitsprävention wird dazu aus historischer und sozialanthropologischer Perspektive verfolgt. Geographisch ist der Band europäisch-vergleichend angelegt, ergänzt durch einen transatlantischen Seitenblick. Dabei stehen Länder wie Deutschland (vom Kaiserreich über die Weimarer Republik und den Nationalsozialismus bis zur DDR und der Bundesrepublik), Großbritannien, die Schweiz oder die USA im Vordergrund.

Untersucht werden nicht nur die zwischenstaatlichen Unterschiede, sondern auch internationale Transfers in der Gesundheitspolitik. Die nationalen Entwicklungspfade des Präventionsdiskurses werden also nicht nur mit Blick auf die Divergenzen, sondern auch auf die Konvergenzen analysiert. Exemplarisch für die Divergenzen stehen beispielsweise die nationalen Leitkrankheiten, nach denen sich die jeweils dominierenden Präventionsstrategien orientierten. Während etwa in den USA der Präventionsdiskurs seit den 1940er Jahren stark auf die Herzkreislaufkrankheiten und die damit verbundenen Ernährungs- und Lebensstilrisiken konzentriert war, standen in Großbritannien die Krebsprävention, insbesondere die Tabakrisiken, später auch der Alkohol im Vordergrund. Deutschland liegt gleichsam in der Mitte. In der Zwischenkriegszeit und im Nationalsozialismus dominierte das Krebsrisiko, in der Nachkriegszeit gewannen die Risiken der Herzkreislaufkrankheiten an Aufmerksamkeit.

Die Präventionsgeschichte spiegelt daneben die zwischenstaatlichen Transfers, die etwa zum internationalen Aufstieg des Risikofaktorenmodells seit den 1950er Jahren und damit zu einer Angleichung nationalstaatlicher Präventionsdiskurse führten. Solche Trends wurden noch unterstützt durch die Arbeit internationaler Organisationen - von den internationalen Kongressen des 19. Jahrhunderts bis zu den supranationalen Organisationen des 20. Jahrhunderts, etwa dem Internationalen Arbeitsamt, der Weltgesundheitsorganisation oder den Organisationen der Europäischen Gemeinschaft, beziehungsweise der Europäischen Union. 
Sie alle bewirkten eine zunehmende Europäisierung und Internationalisierung der Präventionsdiskurse, insbesondere in der zweiten Hälfte des 20. Jahrhunderts (Weindling 1995).

\section{Prävention als Kulturtechnik der Moderne: theoretische Zugänge, zentrale Akteure und ungelöste Paradoxien}

Dass Prävention eine grundlegende Sozial- und Kulturtechnik der Moderne darstellt und die Präventionsgeschichte einen exemplarischen Blick auf die Entwicklung der modernen Gesundheitspolitik eröffnet, ist keine neue Erkenntnis. Schon Auguste Comte hat bekanntlich in seiner 1844 erschienenen Rede über den Geist des Positivismus den positivistisch-forschenden Geist mit der Fähigkeit gleichgesetzt, „zu sehen um vorauszusehen“ - ein Postulat, das später zum positivistischen Leitspruch des Savoir pour prévoir, afin de pouvoir kanonisiert wurde (Comte 1994: 20; Lévy-Bruhl 1931: 14; vgl. auch: Bröckling 2008: 42). Prävention wird hier zum Leitmotiv der sozialwissenschaftlichen Erkenntnisgewinnung und der davon ausgehenden sozialpolitischen Interventionen.

Einen Schritt weiter ging Adolph Wagner, Staatswissenschaftler und prominenter Vertreter der jüngeren historischen Schule der Nationalökonomie, der im ausgehenden 19. Jahrhundert auf den konstitutiven Zusammenhang zwischen Prävention und Moderne hingewiesen hat (zu Wagner: Grimmer-Solem 2003: 172-175). In seinem 1893 erschienenen „Lehr- und Handbuch der politischen Ökonomie“ postulierte Wagner das sogenannte „Gesetz des Vorwaltens des Präventivprincips im entwickelten Rechts- und Culturstaate“ (Wagner 1893: 908-915). Moderne Staaten würden ihr Handeln, so das Argument, zunehmend dem Vorsorgegedanken unterordnen und sich dabei vom „Repressivprinzip“ abwenden. Wagner sprach von einer ,,Veränderung der Art und Weise, in der der Staat seine Tätigkeiten ausführt“ und zwar in dem Sinne, dass „,der Staat zu umfassenden, präventiv Rechtsstörungen verhütenden Maßregeln“ greife (Wagner 1893: 908-912). Den Grund dafür erkannte Wagner im steigenden Komplexitätsgrad moderner Gesellschaften. Es sei effizienter und ökonomischer, gesellschaftliche oder zwischenstaatliche Spannungen und Konflikte durch präventive Verhaltensregeln zu vermeiden als die Konfliktparteien ex post durch Bestrafungen zu sanktionieren. Das „Präventivprinzip“ sah Wagner in sämtlichen Bereichen des staatlichen Handelns im Vormarsch, in der Außen- wie in der Innenpolitik, in der Sozial- wie in der Gesundheitspolitik. 
Auch in neueren sozialwissenschaftlichen und historischen Theoriedebatten wurde wiederholt auf die Wahlverwandtschaft zwischen moderner Staatlichkeit und rationalem Präventionsdiskurs hingewiesen. Schon Reinhart Koselleck hat Prävention als eine auf rationalem Kalkül beruhende Zukunftsbeherrschung und damit als Kernelement eines modernen Rationalisierungsprozesses verstanden - ein Ansatz, der seither vor allem von Lucian Hölscher weiterentwickelt wurde. Danach setzt sich der rationale Zukunftsbegriff im Verlauf des 19. Jahrhunderts gegenüber konkurrierenden Zukunftsmodellen, etwa apokalyptischer, eschatologischer oder astrologischer Provenienz, schrittweise durch. Die Annahme einer rationalisierbaren und damit prognostizierbaren Zukunft öffnete im Verlaufe des 19. Jahrhunderts jenen neuen Wahrnehmungshorizont und jene Handlungsoptionen, auf denen die modernen Präventionspostulate gründeten (Koselleck 1989: 19-34; Hölscher 1999: 3446). Auf die herrschaftstheoretischen Dimensionen dieses Rationalisierungsprozesses haben Soziologen wie Nikolas Rose oder Ulrich Bröckling hingewiesen. In Rückgriff auf Michel Foucault werden präventive Praktiken hier als charakteristisches Merkmal einer Biopolitik der Moderne interpretiert, als eine wissenschaftlich-technologisch fundierte, mit wirkungsmächtigen Rationalitätsansprüchen auftretende Form der Sozial- und Bevölkerungspolitik (Rose 2001; Bröckling 2009: 43-47).

Dass Risikodiskurse in modernen Gesellschaften auch eine sozialkonstitutive Funktion haben können, hat die eingangs erwähnte Mary Douglas verschiedentlich unterstrichen. Sie sieht risk und blame in einem wechselseitig produktiven Verhältnis, mit der Konsequenz, dass soziale Gruppen bestimmte Verhaltensweisen deshalb als riskant ausgrenzen, weil sie damit den normativen Zusammenhalt ihrer Gesellschaft durch präventive Auflagen zu festigen vermögen. Douglas spricht in diesem Zusammenhang auch von der forensischen Funktion der Risikoprävention, weil hier bestimmte Verhaltensweisen quasi kriminalisiert werden. Jedenfalls besitzen Präventionspraktiken in diesem Model sowohl sozialintegrative wie -exklusive Wirkungen (Douglas 1996: 3-21). In vergleichbarer Weise haben auch soziologische Zeitdiagnosen wie jene der Risikogesellschaft oder der Zweiten Moderne (Ulrich Beck) die zentrale Bedeutung von Risiko- und Präventionsdiskursen für das krisenhafte Selbstverständnis westlicher Gesellschaften im ausgehenden 20. und frühen 21. Jahrhundert betont (Beck 2007; vgl. auch Ewald 1993).

Präventionspraktiken lassen sich vor diesem Hintergrund als exemplarischer Gegenstand einer Kulturgeschichte des modernen Gesundheitswesens untersuchen. Dies bezieht sich zunächst auf die Ebene der 
Akteure. Es sind vier Akteursgruppen, von denen die Dynamik der modernen Präventionsgeschichte ausgeht - sie stehen auch in diesem Sammelband im Mittelpunkt.

Zunächst bildet die Gesundheitsprävention ein Anliegen des Staates, beziehungsweise moderner bürokratisch-rationaler Verwaltungsapparate. In der Tradition merkantilistischer und kameralistischer Bevölkerungspolitik entdeckten verschiedene europäische Staaten im Laufe des 19. Jahrhunderts den Vorsorgegedanken als Herrschaftstechnik, um ihre Bevölkerung - als ökonomische und militärische Ressource - möglichst effizient und nachhaltig zu mobilisieren. Der Staat engagierte sich in der präventiven Zukunftsvorsorge nicht zuletzt, um konkurrierende Vorsorgeeinrichtungen, etwa von kirchlichen oder berufsständischen Akteuren, zu verdrängen (Stolleis 2003: 14f.). Dabei war die Legitimität staatlicher Gesundheitsprävention oft besonders hoch. Dies wohl nicht zuletzt, weil der Staat prädestiniert war, den Schutz individuellen Lebens unmittelbar mit dem kollektiven Wohl zu verbinden.

Eine solche etatistische Deutung präventiver Techniken findet sich beispielsweise bei Adolph Wagner, der die Verantwortung für präventives Handeln wie erwähnt beim Staat verortete. Prävention wird dadurch zu einem zentralen Motor einer langfristigen Bürokratisierung; nicht zufällig steht das „Präventionsgesetz“ in Wagners Lehrbuch unmittelbar vor dem weit bekannteren „Gesetz der wachsenden Ausdehnung der Staatstätigkeiten“ (Wagner 1893: 892-912).

Der Präventionsdiskurs geht zweitens von privatwirtschaftlichen Akteuren aus, die in der Forschung oft übersehen werden. $\mathrm{Zu}$ diesen gehören etwa die Versicherungs-, die Pharma- oder die Tabakindustrie, aber auch Gesundheitseinrichtungen wie private Spitäler oder private Krankenkassen. Parallel zum Ausbau der Sozialstaaten lässt sich im 20. Jahrhundert auch ein wachsender Einfluss privater Einrichtungen für die Propagierung präventiver Verhaltensnormen und in Verbindung damit eine Ökonomisierung der Präventionsdiskurse beobachten. Parallel zum Aufstieg des einflussreichen Risikofaktorenmodells wurde der Bereich der Gesundheitsprävention seit der frühen Nachkriegszeit zunehmend kommerzialisiert. Der Vorsorgegedanke wurde damit nicht nur nach marktlogischen und ökonomischen Kriterien umformuliert; zur privatwirtschaftlichen Präventionslogik gehörte auch eine Individualisierung und Subjektivierung der gesundheitsorientierten Verhaltensauflagen (Bröckling 2008: 46f.).

Als dritter Akteur sind zivilgesellschaftliche Vereinigungen zu nennen, etwa die Hygienebewegung des 19. Jahrhunderts oder alternativmedizinische Kreise des 20. Jahrhunderts. In Abgrenzung von einer staatlichen Präventionspolitik lässt sich hier eine stärker lebensweltlich 
verankerten Präventionskultur verfolgen. Präventive Praktiken begründen hier soziale Kohäsions- und Exklusionseffekte, die für das Selbstverständnis zivilgesellschaftlicher Gruppierungen oft prägend waren.

Schließlich liegt präventives Verhalten viertens auch in der Verantwortlichkeit individueller Akteure. Auf diese subjektive Ebene der Präventionsgeschichte hat beispielsweise der britische Soziologe Nikolas Rose hingewiesen, eingebettet in ein historisches Argument. Danach setzte in der zweiten Hälfte des zwanzigsten Jahrhunderts ein Transformationsprozess von staatlichen und zivilgesellschaftlichen $\mathrm{zu}$ liberalindividualistischen Präventionspraktiken ein (Rose 2001: 1-5; vgl. auch: Labisch 1992: 321-325). Diese Subjektivierung der präventiven Verantwortung muss sich nicht in einer Weiterführung staatlicher Vorsorgestrategien erschöpfen; das präventive Selbst, jenes rationale, krankheitsminimierend agierende Subjekt, das in den neueren gesundheitspolitischen Debatten vermehrt beschworen wird, kann sich - ähnlich wie beim Habitus-Modell Pierre Bourdieus - auch dissonant oder in Opposition zu staatlichen Präventionsmodellen entwickeln.

Die hier versammelten Beiträge verstehen sich als Bestandsaufnahme einer noch weitgehend ungeschriebenen Kulturgeschichte der Gesundheitspolitik seit dem ausgehenden 19. Jahrhundert. Sie zielen insbesondere darauf, die grundlegenden Paradoxien präventiver Verhaltensdispositionen in kulturalistischer Perspektive zu erhellen. Zunächst geht es um den Widerspruch zwischen wissenschaftlichem Präventionsanspruch und alltäglichen Präventionspraktiken. Die Präventionsgeschichte ist voll von Beispielen, in denen die präventiven Erwartungen der Experten hoch, die effektiven Resultate dagegen bescheiden waren. Dahinter verbirgt sich eine soziokulturelle Dissonanz der Präventionsvorstellungen. Wissenschaftliche Präventionsmodelle (etwa in der Epidemiologie oder der Sozial- und Präventivmedizin) beziehen sich auf Kollektive, auf die Gesundheit ganzer Populationen. Auf der Ebene von Einzelpersonen dagegen drücken sich präventive Normen meist nur in diffusen Wahrscheinlichkeitsaussagen aus. Die probabilistischen Ansätze der medizinischen Risikoforschung standen deshalb meist in einem konfliktreichen Spannungsverhältnis zu den handlungsleitenden Maximen einzelner Individuen. Anders als der wissenschaftliche Präventionsdiskurs begründeten Individuen ihr vorsorgliches Handeln in der Regel partikularistisch, vor dem Hintergrund ihres subjektiven Erfahrungsraums.

Ein weiteres Paradox, das es im folgenden zu klären gilt, besteht in der nicht-intendierten Vermehrung von Unsicherheit durch den modernen Präventionsdiskurs. Zwar zielten Präventionsbestrebungen auf eine Reduktion der mit Risiken verbundenen Kontingenzen, der Effekt war aber häufig gegenteilig, zumindest in alltagspraktischer Perspektive. 
Durch präventive Sensibilisierungen wurden scheinbar ungefährliche Verhaltensweisen wie Essen, Trinken oder Rauchen unvermittelt zu Gesundheitsrisiken umdefiniert. Mit der Konjunktur der medizinischen Risikoforschung kam es nach dem Zweiten Weltkrieg zu einer Proliferation der medizinischen Risiken und einer Extensivierung entsprechender Präventionsstrategien. Die subjektiven Folgen dieser Entwicklung waren ambivalent. Auf der einen Seite lässt sich eine verstärkte Sensibilisierung beobachten, beispielsweise in den Debatten um die „Kalorienangst“ und um die „Managerkrankheiten“ der frühen Nachkriegszeit (Kury 2010). Seit den 1980er-Jahren zeigen sich aber auch Gegentendenzen und offene Symptome von Präventionskritik und präventivem Überdruss. Die widersprüchlichen Reaktionen zeigen, dass die Zunahme an Präventionsauflagen auf subjektiver Ebene das Potenzial an Verunsicherungen nicht reduziert, sondern eher noch vermehrt hat.

Ein besonderes Augenmerk gilt in den folgenden Beiträgen auch der materiell-körperlichen Dimension der Präventionsgeschichte (vgl. auch: Lengwiler/Beck 2008). Präventionsmaßnahmen zielen in verschiedener Hinsicht auf eine Transformation des Natürlichen: durch Veränderung der äußeren Natur (Umweltfaktoren, Lebensbedingungen) wie auch der inneren Natur (körperliche Zustände, Lebensstile, etc.). Für die Analyse dieser materiellen Dimension nutzen die Beiträge verschiedene innovative theoretische Zugänge, insbesondere die Ansätze der historischen Anthropologie, der Wissenschaftsgeschichte und der Körpergeschichte (exemplarisch: Sarasin 2001: 11-28; Tanner 2004: 123-131).

\section{Vom Vorsorgestaat zum präventiven Selbst: Skizze einer Präventionsgeschichte des 20. Jahrhunderts}

Wie stellen sich die groben Umrisse der Präventionsgeschichte des 20. Jahrhunderts dar? Ausgehend von den hier versammelten Beiträgen sollen im Folgenden einige Entwicklungslinien und Umbrüche des europäischen Präventionsdiskurses skizzenhaft nachgezeichnet werden. Auch wenn der Aufstieg des Vorsorgegedankens oft im 19. und 20. Jahrhundert, als Teil der Rationalisierung und Bürokratisierung der Moderne, angesiedelt wird, reichen die Wurzeln gegenwärtiger präventiver Gesundheitspraktiken durchaus in vor- und frühmoderne Zeiten zurück. So greift etwa der umweltbezogene Präventionsbegriff des modernen Ernährungs- oder des Hygienediskurses auf die frühneuzeitliche Humoralpathologie und ihr komplexes Analogieschema von inneren Säften und äußeren Elementen zurück (z.B. Teleky 1948: 196-201). Insbesondere 
im Populärdiskurs lassen sich humoralpathologische Deutungsmuster wenn auch unter veränderten Vorzeichen - noch bis ins 20. Jahrhundert verfolgen, so etwa bei alternativmedizinischen Ernährungslehren (vgl. dazu die Beiträge von Jakob Tanner und Eberhard Wolff). Auch in institutioneller Hinsicht wurden die Weichen der modernen Gesundheitsprävention bereits in der Frühen Neuzeit gestellt. So etablierte sich das Vorsorgemodell des modernen Versicherungswesens in Großbritannien bereits im 17. und 18. Jahrhundert, zur Absicherung bürgerlicher Einkommen und Vermögenswerte vor frühzeitigen Todesfällen oder zum Schutz vor unternehmerischen Geschäftsrisiken wie Unwettern oder Piraterie im Seehandel (Clark 1999).

Um 1900 wird das Präventionsdenken von einer doppelten gesellschaftlichen Transformation erfasst, die schließlich die Grundlage der modernen Gesundheitsprävention bildet. Auf der einen Seite steht ein epidemiologischer Umbruch, der meist im Begriff der epidemiologischen Transition gefasst wird. Nach dem Ersten Weltkrieg nahmen in den staatlichen Krankheitsstatistiken die chronischen Krankheiten stark $\mathrm{zu}$, unter anderem als Folge der gesteigerten Lebenserwartung und der Zurückdrängung epidemischer Krankheiten durch medizinische und sozialhygienische Maßnahmen. Weil chronische Krankheiten wie Krebs, Herzkreislaufkrankheiten oder Diabetes schwer zu behandeln waren und deren Ursachen - vor allem beim Krebs und den Herzkrankheiten - lange im Dunkeln blieben, verlagerte sich mit der Zunahme der chronischen Krankheiten der medizinische Diskurs zunehmend von kurativen therapeutischen Eingriffen zu präventiven Vorkehrungen (differenziert: Weindling 1992; kritisch zur demographischen bzw. epidemiologischen Transition: Ehmer 2004: 118-127).

Ohne die Bedeutung dieser materiell-biologischen Dimension für die Präventionsgeschichte ganz in Abrede zu stellen, setzen die hier versammelten Beiträge andere Akzente. Denn die epidemiologischen Faktoren allein können den Aufstieg präventiver Deutungs- und Handlungsmuster im Gesundheitswesen nicht erklären. Mindestens so wichtig waren die epistemischen Voraussetzungen für den Aufstieg der Prävention. So beruhte die Wahrnehmung der epidemiologischen Wende auf dem parallelen Aufstieg der Medizinalstatistik und dem gesellschaftlichen Vertrauen, das solchen Quantifizierungen sozialer Realitäten zukam. Erst die Verwendung detaillierter Mortalitäts- und Morbiditätsstatistiken machte die Veränderungen des kollektiven Gesundheitszustandes der Bevölkerung einseh- und verhandelbar für die interessierten zeitgenössischen Beobachter. Dieser Prozess wurde durch verschiedene Akteure vorangetrieben, darunter staatliche Stellen wie die statistischen Ämter oder die öffentlichen gewerbemedizinischen und hygienischen Einrich- 
tungen, aber auch durch Akteure der Privatwirtschaft, insbesondere in Form der Lebensversicherungen und ihrer medizinischen und versicherungsmathematischen Experten (vgl. dazu den Beitrag von Theodore Porter). Nach dem Ersten Weltkrieg konstituierte sich auf dieser epistemischen Grundlage das Feld der medizinischen Risikoforschung, angesiedelt im disziplinären Umfeld der neuen Epidemiologie und der medizinischen Statistik (vgl. Matthews 1995: 97-130).

Hinzu kam, dass der Präventionsgedanke eine wichtige Funktion für die Gesellschafts- und Zivilisationskritik des Fin de siècle und des frühen 20. Jahrhunderts übernahm. Denn als Gegenmodell zur traditionellen kurativen Medizin war das Anliegen der Prävention quasi prädestiniert, zum gesundheitspolitischen Kampfbegriff und zum Spielball sozial- und bevölkerungspolitischer Debatten zu werden. Prävention wurde im ausgehenden 19. Jahrhundert gleichsam zu einem biopolitischen Leitbegriff des modernen Gesundheitswesens (Foucault 1999).

In der Zwischenkriegszeit erlebte der Präventionsdiskurs eine eigentliche Blütezeit. Dafür lassen sich epistemologische wie politische Gründe angeben. In epistemologischer Perspektive konnte der Präventionsgedanke von der Krise der Bakteriologie und ihrer deterministischen Kausalitätsmodelle profitieren. Indem die bakteriologischen Ansätze spätestens im Anschluss an die Spanische Grippe von 1918 zunehmend ihre hegemoniale Stellung in den medizinischen Kausalitätstheorien einbüßten, gewannen umweltbezogene Erklärungen in der Tradition der Hygiene wieder an Beachtung (vgl. dazu: Baldwin 1999: 1-36; Schlich 1999). Wie Silvia Berger in ihrem Beitrag ausführt, wurde dieser Trend noch verstärkt durch eine kritische Selbstreflexion innerhalb der Bakteriologie, die sich seit den 1890er Jahren gegenüber ökologischen, holistischen Krankheitsmodellen schrittweise geöffnet hatte. Dies führte da$\mathrm{zu}$, dass sich nach dem Ersten Weltkrieg die ehemals scharf gezogene Grenze zwischen Hygiene und Bakteriologie weitgehend auflöste; entsprechend gewannen die umweltbezogenen Präventionspostulate der Hygiene auch in breiteren medizinischen Kreisen an Unterstützung.

Hinzu kommt, dass sich die Politisierung des Präventionsdiskurses in der Zwischenkriegszeit weiter fortsetzte und Prävention nun endgültig $\mathrm{zu}$ einem gesundheitspolitischen Schlüsselbegriff avancierte. So gehörten präventive Ansätze zu den Kernanliegen der progressiven Sozialmedizin nach Ende des Ersten Weltkriegs, die sowohl in der sozialdemokratischen Weimarer Republik wie in der Sowjetunion oder in linken Medizinerkreisen Großbritanniens und den USA hoch im Kurs standen, mit nachhaltigen Folgen bis weit über den Zweiten Weltkrieg hinaus (vgl. die Beiträge in: Porter 1997; Moser 2002). Dieser Ansatz wird exemplarisch verkörpert in der Figur Alfred Grotjahns (1869-1931), der 
mit seinem Konzept der Sozialen Hygiene eines der ersten umfassenden Präventionsmodelle schuf, das sowohl sozialpolitische Forderungen (zu Fragen des Wohnungsbaus, der Arbeitsverhältnisse etc.) wie auch individuelle Verhaltensempfehlungen (sportliche Betätigung, gesunde Ernährung, eugenisch begründete Familienplanung etc.) umfasste. Dabei setzte Grotjahn nicht nur bei den klassischen Infektionskrankheiten an, sondern bezog seine Lehre auch auf die neuen Zivilisationskrankheiten, wie den Krebs oder die Herzkreislauferkrankungen. Auch wenn Grotjahns Soziale Hygiene im Nationalsozialismus und der BRD aus politischen Gründen kaum beachtet wurde, entfaltete der Ansatz indirekt, vor allem in der Sowjetunion, den USA und später der DDR, einen langfristig wirksamen Einfluss (vgl. den Beitrag von Ursula Ferdinand). Solche progressiven Ansätze der Gesundheitspolitik fanden in der Zwischenkriegszeit auch Unterstützung in internationalen Organisationen, insbesondere im Internationalen Arbeitsamt (vgl. allg.: Weindling 1995)

Der Begriff der Zivilisationskrankheiten verweist schließlich auf einen kulturhistorischen Faktor, der ebenfalls in der Zwischenkriegszeit zu einer verstärkten Beachtung präventiver Deutungs- und Handlungsmuster beitrug. Mit der Vorstellung von Zivilisations- oder Kulturkrankheiten griff der Präventionsdiskurs auf ältere Formen der dystopischen Gesellschaftskritik zurück und formulierte gleichsam eine medizinisch-pathologische Variante der moralischen Zivilisationskritik des frühen 19. Jahrhunderts. Hinter der Sorge um die neuen Zivilisationskrankheiten verbirgt sich letztlich ein tiefgreifender kulturhistorischer Transformationsprozess, der von Veränderungen der Konsumgewohnheiten im Übergang von Mangel- zu Überflussgesellschaften ausging und damit eine Neukodierung von Ernährungsgewohnheiten einschließlich neuer Pathologien wie der Fettsucht und dem Herzkreislaufrisiko verband. Dieser Diskurs etablierte sich in den 1920er und 30er Jahren und verstärkte sich merklich nach 1945 (Merta 2003). Dabei blieb die Zivilisationskritik nicht bloß ein Experten- oder Elitenphänomen, sondern war alltagskulturell in breiten Schichten verankert. Dies zeigt sich etwa in den höchst erfolgreichen regionalen und nationalen Hygiene- und Gesundheitsausstellungen, etwa der deutschen GeSoLei (Gesundheitspflege, soziale Fürsorge und Leibesübungen) 1926 in Düsseldorf oder der schweizerischen Hyspa (Hygiene, Gesundheit und Sport-Ausstellung) 1931 in Bern (Madarász 2010).

Die neuere Forschung hat sich auch mit der Frage beschäftigt, wie offen der Nationalsozialismus gegenüber präventionsorientierten Gesundheitsmodellen eingestellt war. Zwar stößt man in nationalsozialistischen Quellen durchaus auf Sympathiebekundungen gegenüber alternativmedizinischen und sozialhygienischen Postulaten, insbesondere bei 
der nationalsozialistischen Krebsbekämpfung und der Tabakprävention, außerdem auch in der nationalsozialistischen Ernährungspolitik (exemplarisch: Proctor 1999). Allerdings blieb der nationalsozialistische Präventionsdiskurs weitgehend ein propagandistisches Luftschloss. Spätestens nach Kriegsbeginn wurden die gesundheitspräventiven und ernährungsreformerischen Postulate, angesichts der zunehmenden Rationierungen und der sich ausbreitenden Mangelwirtschaft, Makulatur. Faktisch kam es unter dem Nationalsozialismus - dies konnten neuere Untersuchungen zeigen - zu einer deutlichen Verschlechterung der Ernährungslage und der Gesundheitssituation der breiten Bevölkerung (vgl. Süß 2003).

Nach 1945 zeichnete sich in den gesundheitspolitischen Debatten ein Paradigmenwechsel ab, der auch die präventiven Normensetzungen entscheidend tangierte. Als die Verfassung der Weltgesundheitsorganisation (WHO) 1946 Gesundheit neu nicht mehr als Abwesenheit von Krankheit, sondern aufgrund positiver Qualitäten wie körperlichen, geistigen und sozialen Wohlbefindens definierte, legte sie den Grundstein für eine schrittweise Auflösung der Grenze zwischen Gesundheit und Krankheit. Das Feld der Gesundheitsvorsorge brauchte sich nach dieser Definition nicht mehr auf die Verhinderung pathologischer Einflüsse zu beschränken, sondern konnte nun auf verschiedenste lebensweltliche Sphären erstreckt werden. In den nachfolgenden Jahrzehnten entwickelte die WHO diesen generalisierten Gesundheitsbegriff und die damit verbundenen Präventionskonzepte weiter, insbesondere mit den Konferenzen von Alma Ata (1978/79) und Ottawa (1986). Der Gesundheitsbegriff von 1946 sollte nun insbesondere in den Ländern der Dritten Welt umgesetzt werden. Die WHO hoffte, dass durch ihre Politik sich die Gesundheitsversorgung der unterentwickelten Staaten allmählich den westlichen Industrienationen annäherte. Ein zentrales Mittel dazu bildete die positive Gesundheitsförderung (im Gegensatz zur traditionellen, krankheitsfixierten Vorsorge). Prävention - neu im Sinne einer Gesundheitssicherung (Salutogenese) und nicht mehr der traditionellen Krankheitsverhinderung - wurde damit Teil eines globalisierten Gerechtigkeitsdenkens. Allerdings spielten sich diese Debatten primär auf der Ebene medizinischer Deutungsmuster und politischer Strategiedebatten $\mathrm{ab}$ und erreichten erst in vielfach modifizierter, gebrochener Form den gesundheitspolitischen Alltag.

Die hier versammelten Beiträge zeichnen vor diesem Hintergrund ein ambivalenteres und zugleich differenzierteres Bild der Präventionsgeschichte nach 1945. Die gesundheitlichen Präventionsmodelle waren einerseits einem markanten Verwissenschaftlichungstrend, andererseits einem weiteren Popularisierungsschub ausgesetzt. Die Verwissenschaft- 
lichung des Präventionsdiskurses ist eng an die Rezeption der angelsächsischen Risikofaktoren-Forschung in der Herzkreislaufmedizin gebunden. Dabei verdankt sich der Aufstieg des Risikofaktorenmodells der Ausbreitung kybernetischer, systemanalytischer Organisations- und Steuerungsmodelle, die von den kriegswirtschaftlichen Verwaltungswissenschaften des Zweiten Weltkriegs, vor allem in Großbritannien und den USA, ausging (Lengwiler 2006: 314-320). In der medizinischen Forschung wurde das Konzept der Risikofaktoren vor allem durch die sogenannte Framingham-Studie, einer Langzeituntersuchung der Herzkreislauferkrankungen und der damit korrelierenden Verhaltens- und Umweltfaktoren in Framingham, einer amerikanischen Kleinstadt in Massachusetts, kanonisiert. Seit den 1950er Jahren wurde das Modell auch in verschiedenen europäischen Staaten breit beachtet, mit vielschichtigen Folgen. Zunächst führte das Denken in Risikofaktoren zu einer massiven Ausweitung der potenziellen Gesundheitsrisiken - aufgrund der mathematisch-statistischen Verfahren konnten nun auch kleinste Umweltfaktoren mit Gesundheitsschädigungen korreliert und damit als präventionsrelevant identifiziert werden. Die davon ausgehende Proliferation der identifizierten Gesundheitsrisiken verband sich bereits in der frühen Nachkriegszeit mit einer sprunghaften Extensivierung der Präventionsstrategien. Weiter bildete das Risikofaktorenmodell einen wichtigen Grund dafür, dass sich gegenüber der Sozialen Hygiene die Akzente der präventiven Verhaltensregeln von umwelt- und kontextbezogenen Maßnahmen (Verhältnisprävention) in Richtung individuelles Handeln (Verhaltensprävention) zu verschieben begannen (vgl. dazu den Beitrag von Carsten Timmermann).

Zwar entfaltete sich der Prozess der Individualisierung und Subjektivierung präventiver Praktiken bereits in der Zwischenkriegszeit. Vor allem in bürgerlich-elitären Kreisen, etwa im Umfeld der Lebensreformbewegung, hielt ein solcher Individualisierungstrend schon vor dem Zweiten Weltkrieg Einzug. Darauf verweist etwa der Beitrag von Eberhard Wolff am Beispiel der ernährungsorientierten Bircher-Benner-Privatklinik in Zürich. Auch die sozialhygienische und sozialmedizinische Bewegung um Alfred Grotjahn oder die kritische Bakteriologie betonten zunehmend die individuelle Verantwortlichkeit in Präventionsfragen, um zu verhindern, dass die gesundheitspolitischen Interventionen wirkungslos verpufften. Diese Überlegungen waren jedoch eingebettet in ein allgemeines sozialreformerisches Programm, zu dem auch gesundheitspolitische Maßnahmen und staatliche Interventionen gehörten (vgl. die Beiträge von Silvia Berger und Ursula Ferdinand).

In der Nachkriegszeit setzte nun, initiiert durch den Aufstieg des Risikofaktorenmodells, eine liberale Wende des Präventionsdiskurses ein, 
durch die individuelle Verhaltensempfehlungen auf Kosten institutioneller, sozialstaatlicher Reformen zunehmend in den Vordergrund rückten. Ein treffendes Beispiel dafür liefert der Beitrag von Ulrike Lindner. Die Autorin zeigt darin auf, wie sich in der Schwangerenfürsorge sowohl in Großbritannien wie in Deutschland der Ansatz einer staatlich kontrollierten Gruppenvorsorge zunehmend von einer individuellen Prävention, begleitet durch die niedergelassenen Ärzte, abgelöst wurde. Solche Veränderungsprozesse standen in enger Wechselwirkung mit anderen gesellschaftlichen Individualisierungstrends, etwa im Kontext der sich ausbreitenden Konsum- und Freizeitgesellschaft. Individualistische Lebensstile öffneten neue Felder der Prävention und wurden ihrerseits zunehmend präventionsorientiert umformuliert. Ein Beispiel dafür ist der Aufstieg von freizeitsportlichen Aktivitäten wie etwa des Joggings, der sich in den 1970er Jahren nicht zuletzt dem Hinweis auf die gesundheitsfördernde Wirkung des Sports verdankte. Die Geschichte des Breitensports zeigt darüber hinaus, dass das Anliegen der Krankheitsvorsorge in der Nachkriegszeit zunehmend ergänzt und überlagert wurde durch das Modell der Gesundheitsförderung, ein Prozess, der dem Präventionsdiskurs insgesamt einen zusätzlichen, bis heute anhaltenden Auftrieb verliehen hat (vgl. dazu den Beitrag von Tobias Dietrich).

Solche Individualisierungsprozesse wurden noch befördert durch zunehmend professionelle Kampagnen zur Popularisierung der Gesundheitsprävention. Wie Virginia Berridge in ihrem Beitrag aufzeigt, nutzte die Sozial- und Präventivmedizin der frühen Nachkriegszeit für ihr Popularisierungsanliegen die Erkenntnisse der zeitgenössischen Propaganda- und Marktforschung. Mit dem Aufstieg professioneller Marketingmethoden in der Gesundheitsprävention veränderte sich auch der Status des angesprochenen Publikums: Aus dem staatsbürgerlichen Individuum wurde neu ein Konsument beziehungsweise eine Konsumentin präventiver Verhaltensempfehlungen.

Die Breitenwirkung des Präventionsdiskurses konnte nach 1945 ähnlich wie bereits in der Zwischenkriegszeit - von vorsorgeorientierten alltagskulturellen Dispositionen profitieren. Dies zeigt sich etwa an der populären Wahrnehmung der Herzkreislaufkrankheiten, die in den 1950er und 60er Jahren im deutschsprachigen Raum unter dem Begriff der Managerkrankheiten diskutiert wurden. Wie Patrick Kury jüngst aufgezeigt hat, verwob diese populäre Krankheitsdiagnose geschickt verbreitete Modernisierungs- und Amerikanisierungsängste und formte daraus eine pathologisierend grundierte Zivilisationskritik (Kury 2010). Dass solche Deutungsmuster auf fruchtbaren Boden fielen, hängt mit dem beschleunigten kulturellen und gesellschaftlichen Wandel der Nachkriegszeit zusammen. Die Veränderungen der Erwerbswelt mit der 
Automatisierung der Industriearbeit und dem Aufstieg des Dienstleistungssektors sowie die Transformation der Konsumgewohnheiten im Übergang von der kriegsbedingten Mangelwirtschaft zur Überflussgesellschaft der Nachkriegsjahrzehnte wurden im populären Diskurs vor allem auf die damit verbundenen Gesundheitsrisiken - Stress, Bewegungsmangel oder Übergewicht, beziehungsweise die davon ausgehenden Herzkreislaufkrankheiten - reduziert (vgl. dazu den Beitrag von Jeannette Madarász in diesem Band).

Als Folge dieser vielschichtigen Individualisierungs- und Popularisierungsprozesse wurden präventive Ansätze nach 1945 zunehmend zu inkorporierten Elementen subjektiver Körper- und Gesundheitsvorstellungen. Die historische Entwicklung präventiver Verhaltensnormen gleicht deshalb einem langfristigen Interiorisierungsprozess, der in seiner longue durée von den etatistischen Vorsorgemodellen spätabsolutistischer Staaten bis zum individualistischen Leitbild des präventiven Selbst - einem rationalen Subjekt, das sich kontinuierlich beobachtet und sein Verhalten nach gesundheitlichen Kriterien selbst diszipliniert reicht.

Die jüngste Phase der Präventionsgeschichte setzte in den 1990er Jahren ein und ging vom Aufstieg biomedizinischer und humangenetischer Ansätze im medizinischen Fachdiskurs aus. Der Beitrag von Martin Döring illustriert diesen Umbruch exemplarisch am Konzept des metabolischen Syndroms, das sich in den letzten Jahren als zentrales Erklärungsmodell der Herzkreislaufkrankheiten etabliert hat. Das Konzept betont die Bedeutung des Zellstoffwechsels und der Gewebebeschaffenheit für die Entstehung von Bluthochdruck, Fettleibigkeit, Thromboserisiken und Diabetes (Typ 2). Die Stoffwechselfunktionen wiederum werden einerseits auf genetische Prädispositionen, andererseits auf umweltbedingte Einflüsse zurückgeführt; beide Faktoren zusammen sind in einem hochkomplexen Syndrommodell vereint. Döring verweist einerseits auf die unsichere Kohärenz der medizinischen Terminologie und führt andererseits deren verblüffenden Erfolg gerade auf die Vagheit und Offenheit des Syndromkonzepts zurück. Für den Präventionsbegriff wichtig ist, dass die Risiken chronischer Krankheiten mit der Diagnose eines metabolischen Syndroms vervielfacht werden und damit auch die Ansatzpunkte für gesundheitsorientierte Verhaltensempfehlungen weiter zunehmen. Sie erstrecken sich nun endgültig auf die Ebene normaler alltäglicher Verhaltensweisen - eine Pathologisierung des lebensweltlichen Alltags, die die Grenze zwischen krank- und gesundmachenden Handlungen vollständig verwischt.

$\mathrm{Zu}$ demselben Schluss kommen auch die Beiträge von Jakob Tanner und Robert Aronowitz. Beide konstatieren, dass die intensivierte medi- 
zinische Risikoforschung zu einer massiven Proliferation der Gesundheitsrisiken geführt habe - Tanner bezieht sich auf die Debatte um krankhafte Formen des Übergewichts (Adipositas), Aronowitz auf die Brustkrebsforschung. Die Diskussion um Gesundheitsrisiken habe, so Tanner, inzwischen eine „pandemische Qualität“ erreicht, mit entsprechenden Verunsicherungen auf Seiten der risikoexponierten Bevölkerung. Aronowitz weist darüber hinaus darauf hin, dass anstelle des einst klaren Gegensatzes zwischen gesund und krank heute ein Kontinuum zwischen schwacher und starker Risikoexposition entstanden ist. Damit verbunden ist eine massive Zunahme potenziell gefährdeter Patientinnen und Patienten, die sich nun nicht mehr entlang der Trennlinie zwischen gesund und krank einordnen können, sondern mit einer Fülle präventionsorientierter Verhaltensauflagen konfrontiert werden, auch wenn sie selber noch gar keine Krankheitssymptome aufweisen.

Solche risikobasierte Krankheitsdiagnosen zeitigen für Patientinnen und Patienten durchaus paradoxe Verhaltenseffekte. Diesen Punkt illustriert insbesondere der Beitrag von Jörg Niewöhner, der sich unter anderem mit der Wahrnehmung des metabolischen Syndroms durch Patientinnen und Patienten beschäftigt. Denn die Genetisierung der medizinischen Diagnosen engt letztlich den ärztlichen Behandlungsspielraum sowie die Handlungsfähigkeiten von Patientinnen und Patienten deutlich ein, was indirekt die Legitimität der präventiven Verhaltensauflagen untergräbt. Auch ist es ein schwieriges Unterfangen, so Niewöhner, angesichts der hohen Komplexität biomedizinischer Erklärungsmodelle überhaupt nachvollziehbare präventive Verhaltensempfehlungen zu formulieren.

Auch Robert Aronowitz betont die widersprüchlichen Folgen aktueller Präventionsdiskurse für die betroffenen Individuen. Auf der einen Seite lässt sich ein steigender Präventionsdruck diagnostizieren, verbunden mit einer akzentuierten Risikoangst. Auf der anderen Seite finden sich aber auch Anzeichen einer Art kognitiver Erschöpfung (eines risk fatigue). Die subjektiven Reaktionen auf die jüngste Proliferation der Gesundheitsrisiken pendeln damit zwischen Sensibilisierung und Banalisierung der Risikopotenziale hin und her.

Die intendierte Pathologisierung vermeintlich normaler Verhaltensweisen entpuppt sich damit in der medizinischen Praxis letztlich als Phyrrussieg. In den USA hat die Pathologisierung des Übergewichts inzwischen dazu geführt, dass das Land heute mehr Übergewichtige (und damit potenziell Adipositas-Gefährdete) zählt als Einkommenssteuerpflichtige (auf Bundesebene; vgl. The Economist, 23.1.2010, S. 43). Damit ist aus der pathologischen Anomalie endgültig eine neue Norma- 
lität geworden. Der moderne Präventionsdiskurs hat mit anderen Worten begonnen, seine eigenen Existenzgrundlagen zu untergraben.

\section{Bibliographie}

Aronowitz, Robert A. (1998): Making Sense of Illness: Science, Society, and Disease, Cambridge: Cambridge University Press.

Baldwin, Peter (1999): Contagion and the State in Europe, 1830-1930, Cambridge: Cambridge University Press.

Beck, Ulrich (2007): Weltrisikogesellschaft: auf der Suche nach der verlorenen Sicherheit, Frankfurt a.M.: Suhrkamp.

Bröckling, Ulrich (2008): „Vorbeugen ist besser ... Zur Soziologie der Prävention“. In: Behemoth. A Journal on Civilisation 1, S. 38-48.

Clark, Geoffrey (1999): Betting on Lives: The Culture of Life Insurance in England 1695-1775, Manchester: University Press.

Comte, Auguste (1994): Rede über den Geist des Positivismus, Hamburg: Felix Meiner.

Douglas, Mary/Wildavsky, Aaron (1983): Risk and Culture. An essay on the selection of technological and environmental dangers, Berkeley: University of California Press.

Douglas, Mary (1996): Risk and blame. Essays in cultural theory, London: Routledge.

Ehmer, Josef (2004): Bevölkerungsgeschichte und Historische Demographie 1800-2000, München: Oldenbourg.

Ewald, François (1993): Der Vorsorgestaat, Frankfurt a.M.: Suhrkamp.

Foucault, Michel (1999): „Vorlesung vom 17. März 1976“. In: Michel Foucault (Hg.), Verteidigung der Gesellschaft. Vorlesungen am Collège de France (1975-76), Frankfurt a.M.: Suhrkamp, S. 276-305.

Grimmer-Solem, Erik (2003): The rise of historical economics and social reform in Germany 1864-1894, Oxford: Clarendon Press.

Hölscher, Lucian (1999): Die Entdeckung der Zukunft, Frankfurt a.M.: Fischer.

Koselleck, Reinhard (1989): Vergangene Zukunft. Zur Semantik geschichtlicher Zeiten, Frankfurt a.M.: Suhrkamp.

Kury, Patrick (2010): „Zivilisationskrankheiten an der Schwelle zur Konsumgesellschaft. Das Beispiel der Managerkrankheit in den 1950er und 1960er Jahren“. In: Petra Overath et al. (Hg.), Die vergangene Zukunft Europas. Kulturwissenschaftliche Analysen von demografischen Prognosen und Wissensordnungen, Köln: Böhlau. (Im Druck) 
Labisch, Alfons (1992): Homo hygienicus: Gesundheit und Medizin in der Neuzeit, Frankfurt a.M.: Campus.

Lengwiler, Martin (2006): Risikopolitik im Sozialstaat. Die schweizerische Unfallversicherung 1870-1970, Köln: Böhlau.

Lengwiler, Martin/Beck, Stefan (2008): „Historizität, Materialität und Hybridität von Wissenspraxen. Die Entwicklung europäischer Präventionsregime im 20. Jahrhundert". In: Geschichte und Gesellschaft, Zeitschrift für historische Sozialwissenschaft 33, S. 489-523.

Lévy-Bruhl, Lucien (1931): Le surnaturel et la nature dans la mentalité primitive, Paris: Alcan.

Madarász, Jeannette (2010): „Die Prävention chronischer Erkrankungen des Herz-Kreislauf-Systems in Deutschland: der Risikofaktor Übergewicht“. In: Christoph Heintze (Hg.), Public Health, Übergewicht und Prävention, Weinheim: Juventa Verlag. (Im Druck)

Matthews, J. Rosser (1995): Quantification and the Quest for Medical Certainty, Princeton: Princeton University Press.

Merta, Sabine (2003): Wege und Irrwege zum modernen Schlankheitskult. Diätkost und Körperkultur als Suche nach neuen Lebensstilformen 1880-1930, Wiesbaden: Franz Steiner Verlag.

Moser, Gabriele (2002): „Im Interesse der Volksgesundheit... “ Sozialhygiene und öffentliches Gesundheitswesen in der Weimarer Republik und der frühen SBZ/DDR. Ein Beitrag zur Sozialgeschichte des deutschen Gesundheitswesens im 20. Jahrhundert, Frankfurt a.M.: VAS.

Porter, Dorothy (1997) (Hg.): Social Medicine and Medical Sociology in the Twentieth Century, Amsterdam: Rodopi.

Proctor, Robert N. (1999): The Nazi War on Cancer, Princeton: Princeton University Press.

Rose, Nikolas (2001): „The Politics of Life Itself“. In: Theory, Culture \& Society 18/6, S. 1-30.

Sarasin, Philipp (2001): Reizbare Maschinen. Eine Geschichte des Körpers 1765-1914, Frankfurt a.M.: Suhrkamp.

Schlich, Thomas (1999): „Einführung: Die Kontrolle notwendiger Krankheitsursachen als Strategie der Krankheitsbeherrschung im 19. und 20. Jahrhundert“. In: Christoph Gradmann/Thomas Schlich (Hg.), Strategien der Kausalität: Konzepte der Krankheitsverursachung im 19. und 20. Jahrhundert, Pfaffenweiler: Centaurus, S. 328.

Stolleis, Michael (2003): Geschichte des Sozialrechts in Deutschland. Ein Grundriss, Stuttgart: Lucius \& Lucius. 
Süß, Winfried (2003): Der ,,Volkskörper“ im Krieg. Gesundheitspolitik, Gesundheitsverhältnisse und Krankenmord im nationalsozialistischen Deutschland 1939-1945, München: Oldenbourg.

Tanner, Jakob (2004): Historische Anthropologie zur Einführung, Hamburg: Junius.

Teleky, Ludwig (1948): History of Factory and Mine Hygiene, New York: Columbia University Press.

Wagner, Adolph (1983): Grundlegung der politischen Ökonomie, erster Teil: Grundlagen der Volkswirtschaft, zweiter Halbband, Leipzig: C.F. Winter.

Weindling, Paul (1992): „From Infectious to Chronic Diseases. Changing Patterns of Sickness in the Nineteenth and Twentieth Century“. In: Andrew Wear (Hg.), Medicine in Society, Cambridge: Cambridge University Press, S. 303-316.

Weindling, Paul (1995) (Hg.): International health organisations and movements, 1918-1939, Cambridge: Cambridge University Press. 